\title{
Lactoferrin attenuates fatty acid-induced lipotoxicity via Akt signaling in hepatocarcinoma cells
}

\begin{tabular}{|r|l|}
\hline Journal: & Biochemistry and Cell Biology \\
\hline Manuscript ID & bcb-2015-0014.R1 \\
\hline Danuscript Type: & Article \\
\hline Complete List of Authors: & $\begin{array}{l}\text { Morishita, Satoru; Lion Corpration, ; The University of Tokyo, } \\
\text { Tomita, Keiko; The University of Tokyo, } \\
\text { Ono, Tomoji; Lion Corpration, } \\
\text { Murakoshi, Michiaki; Lion Corpration, } \\
\text { Saito, Kenji; The University of Tokyo, } \\
\text { Sugiyama, Keikichi; Lion Corpration, ; Ritsumeikan University, } \\
\text { Nishino, Hoyoku; Kyoto Prefectural University of Medicine, } \\
\text { Kato, Hisanori; The University of Tokyo, }\end{array}$ \\
\hline Keyword: & \begin{tabular}{l} 
lactoferrin, steatohepatitis, Akt, apoptosis, cytotoxicity \\
\hline
\end{tabular} \\
\hline
\end{tabular}


Title page

Lactoferrin attenuates fatty acid-induced lipotoxicity via Akt signaling in hepatocarcinoma cells

Satoru Morishita ${ }^{1,2}$; Keiko Tomita ${ }^{2}$; Tomoji Ono ${ }^{1,3}$; Michiaki Murakoshi, ${ }^{1,4}$ Kenji Saito ${ }^{2}$; Keikichi Sugiyama $^{1,5}$; Hoyoku Nishino ${ }^{4}$ and Hisanori Kato ${ }^{2}$

${ }^{1}$ Research and Development Headquarters, Lion Corporation, 100 Tajima, Odawara, Kanagawa 256-0811, Japan; "2“Food for Life,” Organization for Interdisciplinary Research Projects, The University of Tokyo, 1-1-1 Yayoi, Bunkyo-ku, Tokyo 113-8657, Japan; ${ }^{3}$ Advanced Medical Research Center, Yokohama City University, 3-9 Fukuura, Kanazawa-ku, Yokohama, Kanagawa 236-0004, Japan; ${ }^{4}$ Kyoto Prefectural University of Medicine, Kawaramachi-Hirokoji, Kamigyou-ku, Kyoto 602-0841, Japan; ${ }^{5}$ Research Organization of Science and Engineering, Ritsumeikan University, 1-1-1 Nojihigashi, Kusatsu, Shiga 525-8577, Japan.

To whom correspondence should be addressed:

Hisanori Kato, "Food for Life", Organization for Interdisciplinary Research Projects, The University of Tokyo, 1-1-1 Yayoi, Bunkyo-ku, Tokyo 113-8657, Japan

Phone: +81-3-5841-1607; Fax: +81-3-5841-1607; E-mail: akatoq@mail.ecc.u-tokyo.ac.jp

Running title: Lactoferrin attenuates lipotoxicity in hepatocarcinoma cells

No specific grant was received from any funding agency in the public, commercial, or not-for-profit sectors with regards to this study. 
Abstract and keywords

Abstract

Nonalcoholic fatty liver disease (NAFLD) describes a spectrum of lesions ranging from simple steatosis to non-alcoholic steatohepatitis (NASH). The excess influx of fatty acids (FAs) into the liver is recognized as a main cause of simple steatosis formation and progression to NASH. Recently, administration of lactoferrin (LF), a glycoprotein present in milk, was suggested to prevent NAFLD development. However, the effect of LF on the contribution of FA to NAFLD development remains unclear. In this study, the effects of LF on FA mixture (FAm)-induced lipotoxicity using human hepatocarcinoma G2 cells were assessed. FAm significantly decreased cell viability and increased intracellular lipid accumulation, whereas LF significantly recovered cell viability without affecting lipid accumulation. FAm-induced lactic dehydrogenase (LDH) and caspase-3/7 activities were significantly decreased by LF and SP600125, a c-Jun N-terminal kinase (JNK) specific inhibitor. We also found that LF added to FAm-treated cells induced Akt phosphorylation which contributed to inhibition of JNK signaling pathway-dependent apoptosis. Akt inhibitor VIII, an allosteric Akt inhibitor, significantly attenuated the effect of LF on LDH activity and abrogated the ones on cell viability and caspase-3/7 activity. In summary, the present study has revealed that LF has a protective effect on FAm-induced lipotoxicity in a HepG2 model of NAFLD and identified the activation of the Akt signaling pathway as a possibly major mechanism.

Key words: lactoferrin, steatohepatitis, Akt, apoptosis, cytotoxicity 
Introduction

Nonalcoholic fatty liver disease (NAFLD) closely correlated to obesity and insulin resistance is considered a hepatic manifestation of metabolic syndrome (Eguchi et al. 2006; Nehra et al. 2001). Moreover, NAFLD describes a spectrum of lesions ranging from simple steatosis to steatosis combined with severe hepatic injury, such as in non-alcoholic steatohepatitis (NASH), which is characterized by cell death and inflammation. The prevalence of NAFLD has continued to increase worldwide, with about 10\% of patients initially diagnosed with NASH, which often progresses to end-stage liver diseases, such as cirrhosis or hepatocellular carcinoma. However, the exact molecular mechanisms of the pathogenesis of NAFLD remain unclear, while effective therapeutic and preventive strategies are limited (e.g., weightreducing nutritional regimens for overweight and obese subjects). Current research supports the "multiple-hit model" in the pathogenesis of NAFLD. As the "first hit," insulin resistance causes an increase in serum fatty acid (FA) concentrations and excess influx of FAs into the liver, resulting in simple steatosis (Tilg and Moschen 2010). Lipotoxicity, such as direct lipid cytotoxicity, dysregulated hepatocyte apoptosis, and inflammation, and so on represents the "subsequent hit," which leads to hepatic dysfunction, resulting in progression to NASH (Tilg and Moschen 2010). In particular, an increased FA supply to the liver has been strongly suggested to play a major role in hepatic lipotoxicity, as indicated by the close correlation between elevated serum FA concentrations and NAFLD severity (Nehra et al. 2001; Zhang et al. 2014). Therefore, in vitro models of FA-overloaded conditions have been created using human hepatic cells to clarify the mechanisms of NAFLD pathogenesis (Chavez-Tapia et al. 2012; Gomez-Lechon et al. 2007; Lin et al. 2007; Wu et al. 2008).

Lactoferrin (LF) is a well-known multifunctional glycoprotein with anti-bacterial, anti-viral, immunostimulatory, antioxidant, and cancer-preventive potentials (Harmsen et al. 1995; Sekine et al. 1997; Shoji et al. 2007; Tomita et al. 1991; Zimecki et al. 1998). Because bovine LF is a natural component of breast milk, it is considered safe and has been classified as "generally recognized as safe" by the US Food and Drug Administration and approved as a food additive in Japan. In a previous study, we found that orally administered enteric-coated bovine LF in the form of a tablet significantly reduced 
visceral fat accumulation, which is known to be strongly associated with symptoms of metabolic syndrome, as described in a double-blind clinical trial (Ono et al. 2010). As possible mechanisms, antiadipogenic actions on pre-adipocytes and cell lines, and lipolytic actions of LF on mature adipocytes have been suggested (Moreno-Navarrete et al. 2009; Ono et al. 2013; Ono et al. 2011; Yagi et al. 2008). Also, LF reportedly improved plasma lipid profiles and hepatic triglyceride accumulation in an animal study of mice fed normal diets (Morishita et al. 2013; Takeuchi et al. 2004). Moreover, a recent study reported that LF administration exhibited beneficial effects on serum lipid profiles and hepatic triglyceride accumulation as the "first hit" in a high-fructose corn syrup-induced NAFLD model (Li and Hsieh 2014). In this report, it is also demonstrated that LF contributes to the inhibition of the hepatic inflammatory cytokines as a "subsequent hit" by scavenging lipopolysaccharides from the circulation. Although fructose directly enters the glycolytic pathway and causes an increase in hepatic-free FA accumulation, the effects of LF on the contribution of FA accumulation to NAFLD development have not been fully investigated. Therefore, the aim of this study was to clarify the direct effect of LF administration on a FAinduced in vitro model of NAFLD and identify the underlying mechanisms of LF. 
Materials and Methods

\section{Materials}

Bovine LF was purchased from FrieslandCampina (Amersfoort, The Netherlands). According to the certificate of analysis, typical protein purity was 98\%. Pepsin-digested LF (pdLF) was prepared as described previously (Ono et al. 2011). FA-free BSA, oleic acid, palmitic acid, linoleic acid, linolenic acid, and arachidonic acid were purchased from Sigma-Aldrich (St. Louis, MO, USA). SP600125, a c-Jun N-terminal kinase (JNK) inhibitor and tunicamycin were purchased from Wako Pure Chemical Industries, Ltd. (Tokyo, Japan). Akt inhibitor VIII was purchased from Sigma-Aldrich. Primary antibodies to Akt, phospho-Akt, and $\beta$-actin, and secondary horseradish peroxidase-conjugated anti-rabbit IgG antibody were purchased from Cell Signaling Technology, Inc. (Beverly, MA, USA).

\section{Preparation of FA mixture (FAm)}

FA mixture (FAm) was prepared as previously reported (Lin et al. 2007). Oleic acid (C18:1), palmitic acid (C16:0), linoleic acid (C18:2), linolenic acid (C18:3), and arachidonic acid (C20:4) were mixed at a ratio of 25:40:15:15:5 in $0.1 \mathrm{~N} \mathrm{NaOH}$ solution at $70^{\circ} \mathrm{C}$ and then mixed with $2 \%$ FA-free BSA solution $(1: 1)$ at $55^{\circ} \mathrm{C}$ and incubated for $10 \mathrm{~min}$. The FA/BSA complex solution (50 $\mathrm{mM}$ as FAm) was filtered and sterilized through a $0.45-\mu \mathrm{m}$ pore membrane filter and stored at $-20^{\circ} \mathrm{C}$. The FA/BSA complex was dissolved and incubated at $55^{\circ} \mathrm{C}$ in a water bath for $10 \mathrm{~min}$ before use.

\section{$\underline{\text { Cell culture }}$}

Hepatocarcinoma G2 (HepG2) cells were cultured in DMEM (Sigma-Aldrich) supplemented with 10\% FBS (Cell Culture Bioscience, Tokyo, Japan), $100 \mathrm{U} / \mathrm{mL}$ penicillin, and $100 \mu \mathrm{g} / \mathrm{mL}$ streptomycin (SigmaAldrich), and maintained at $37^{\circ} \mathrm{C}$ in a humidified atmosphere of $5 \% \mathrm{CO}_{2}$. The cells were subcultured in 6well plates for western blot analysis, in 24-well plates for the quantitation of intracellular lipids, and in 96-well plates for analyses of lipotoxicity-related outcomes. After subculturing, the cells were grown to 
$70 \%$ confluence and starved in serum-free DMEM. After $24 \mathrm{~h}$, the HepG2 cells were treated with FAm for various time periods $(2,4,8$, and $20 \mathrm{~h})$. LF $(5-100 \mu \mathrm{g} / \mathrm{mL})$, pdLF $(100 \mu \mathrm{g} / \mathrm{mL})$, or SP600125 $(50 \mu \mathrm{M})$ were simultaneously added to the FAm-treated HepG2 cells. When appropriate, Akt inhibitor VIII (50 $\mu \mathrm{M})$ was simultaneously added to the HepG2 cells treated with FAm and LF for $2.5 \mathrm{~h}$ and then the cells were cultured without Akt inhibitor VIII for 17.5 h. To induce endoplasmic reticulum (ER) stress, tunicamycin (2 or $10 \mu \mathrm{g} / \mathrm{mL}$ ) was added simultaneously to the HepG2 cells with LF and then incubated for $20 \mathrm{~h}$.

\section{Oil-red O staining}

The cells were washed twice with ice-cold PBS and fixed in 10\% formalin for $60 \mathrm{~min}$. After fixation, the cells were stained with Oil-Red O solution for $15 \mathrm{~min}$ at room temperature. After staining, the cells were washed twice with distilled water. Intracellular lipid droplets were observed under a phase-contrast microscope.

\section{Intracellular fat quantitation}

Intracellular fat accumulation in HepG2 cells was quantified as reported previously (Avramoglu et al. 1995). At $20 \mathrm{~h}$ after FAm treatment, cells were washed two times with $1 \mathrm{~mL}$ of ice-cold PBS and the intracellular lipids were extracted with $1 \mathrm{~mL}$ heptane-isopropanol $(3: 2, \mathrm{v} / \mathrm{v})$ at room temperature for 30 min. Concentrated lipids were reconstituted in 2-propanol and the TG concentrations were analyzed using the Triglyceride E-Test Wako lipid assay kit (Wako Pure Chemical Industries, Ltd.). Cell protein was solubilized using $1 \mathrm{~mL}$ of $0.1 \mathrm{~N} \mathrm{NaOH}$ and quantified by the Bradford method.

\section{Analysis of lipotoxicity-related outcomes}

Cell culture supernatants were used to analyze lactic dehydrogenase (LDH) activity and cells were used to measure caspase-3/7 activity and cell viability. LDH activity was analyzed using the LDH Cytotoxic test 
kit (Wako Pure Chemical Industries, Ltd.). Caspase-3/7 activity was analyzed using the Caspase-Glo ${ }^{\circledR} 3 / 7$ Assay (Promega Corporation, Madison, WI, USA) or the Apo-ONE ${ }^{\mathrm{TM}}$ Homogeneous Caspase-3/7 Assay (Promega Corporation). Resazurin reduction activity, as the outcome of cell viability, was analyzed using the CellTiter-Blue ${ }^{\mathrm{TM}}$ Cell Viability Assay (Promega Corporation). All measurements were performed with a Spectra MAX spectrophotometer (Life Technologies, Grand Island, NY, USA) following the manufacturer's protocol.

\section{Western blot analysis}

Whole cell lysates were prepared in lysis buffer (10 mM Tris, pH 7.4, $150 \mathrm{mM} \mathrm{NaCl}, 10 \mathrm{mM} \mathrm{Na}_{4} \mathrm{P}_{2} \mathrm{O}_{7} \cdot 10$ $\mathrm{H}_{2} \mathrm{O} 1.0 \mathrm{mM}$ EDTA, $1.0 \mathrm{mM}$ EGTA, 0.5\% Nonidet P-40, and 1\% Triton-X) containing $0.2 \%$ protease inhibitors (Sigma-Aldrich) and 1\% phosphatase inhibitors (Nacalai Tesque, Inc., Kyoto, Japan). The protein concentrations in the extracts were determined using the Bio-Rad Protein Assay kit (Bio-Rad Laboratories, Inc., Hercules, CA, USA). Typically, 10-20 $\mu \mathrm{g}$ of protein from whole cell lysates were loaded on $8 \%$ sodium dodecyl sulfate-polyacrylamide electrophoresis gels. The separated proteins were transferred to an Immobilon-P membrane (EMD Millipore Corporation, Bedford, MA, USA), which was blocked for $1 \mathrm{~h}$ in blocking buffer (TBS with $0.05 \%$ Tween 20 containing 3\% BSA), and then incubated with primary antibody diluted in TBS with $0.1 \%$ Tween 20 containing $5 \%$ BSA or at $4{ }^{\circ} \mathrm{C}$ overnight. After washing, the blot was incubated with the secondary antibody for $1 \mathrm{~h}$ in TBS buffer. Then, the membrane was washed and target protein images were captured using a Light-Capture instrument (AE6981; ATTO Co., Ltd., Tokyo, Japan) with the ECL Western Blotting Detection System (GE Healthcare, Waukesha, WI, USA). Quantification of Akt and phospho-Akt immunoreactive areas was performed using a Light-Capture instrument. Primary antibodies were diluted to 1:1000 and secondary antibodies were diluted to 1:5000.

\section{$\underline{\text { Statistical analysis }}$}

Data are presented as the means \pm standard deviations. Basically, data were compared using one-way 
ANOVA and the post-hoc Tukey-Kramer test. Time-course data were compared using two-way ANOVA and the post-hoc Student's $t$-test with Bonferroni corrections. Lipid accumulation data were compared using the Dunnett test. A probability $(p)$ value $<0.05$ was considered statistically significant. Data were analyzed using SPSS ver. 19 statistical software (IBM-SPSS, Inc., Chicago, IL, USA). 
Results

Effects of LF on cell viability and lipid accumulation in the FAm-induced NAFLD model

FAm treatment $(1 \mathrm{mM})$ significantly decreased cell viability, whereas LF $(100 \mu \mathrm{g} / \mathrm{mL})$ simultaneously added with FAm recovered cell viability, but pdLF (100 $\mu \mathrm{g} / \mathrm{mL})$ did not (Fig. 1A). Significant effects of LF on cell viability were observed at concentrations of $20-100 \mu \mathrm{g} / \mathrm{mL}$ (Fig. 1B). Intracellular lipid accumulation of HepG2 cells was significantly increased by FAm treatment (Table 1). LF had no effect on intracellular lipid accumulation. Microscopic observation confirmed that LF treatment $(100 \mu \mathrm{g} / \mathrm{mL})$ obviously inhibited the FAm-induced cell death without affecting the lipid accumulation (Fig. 1C, D and E).

\section{Effect of LF on FAm-induced cytotoxicity and apoptosis}

LDH activity and caspase-3/7 activity were significantly increased at $20 \mathrm{~h}$ after FAm treatment (Fig. 2). FAm-induced LDH activity was significantly inhibited by LF $(5-100 \mu \mathrm{g} / \mathrm{mL})$ in a dose-dependent manner. The LF treatment $(100 \mu \mathrm{g} / \mathrm{mL})$ exhibited a $42 \%$ decrease in LDH activity compared with FAm-treated group, but didn't completely inhibit the FAm-induced LDH activity compared with no-treated group (Fig. 2A). The effect of LF on caspase-3/7 activity showed a similar result as that on LDH activity. However, caspase-3/7 activity was completely inhibited by $20 \mu \mathrm{g} / \mathrm{mL}$ LF treatment compared with no-treated group (Fig. 2C). Since apoptosis induced by caspase-3 via the JNK signaling pathway was proposed to play a primary role in FAm-induced lipotoxicity, inhibition experiments were performed. SP600125 (50 $\mu \mathrm{M})$, a JNK-specific inhibitor, exhibited significant inhibition of FAm-induced LDH and caspase-3/7 activities (Fig. 2B, D). The results of the time-course analysis showed that FAm treatment significantly increased LDH activities at all-time points $(2,4$, and $8 \mathrm{~h}$ ) as compared to the untreated group (Fig. 3A). LF significantly inhibited the increase in FAm-induced LDH activity at $4 \mathrm{~h}(21 \%$ decrease), but not at 2 and $8 \mathrm{~h}(16 \%$ and 14\% decrease, respectively) (Fig. 3A). A significant increase in caspase-3/7 activity was observed from $4 \mathrm{~h}$ after FAm treatment, whereas significant inhibitory effects of LF were observed at $8 \mathrm{~h}$ 
(46\% decrease) (Fig. 3B). JNK phosphorylation by FAm treatment didn't be detected at 2 and $4 \mathrm{~h}$ (data not shown), while FAm treatment slightly increased JNK phosphorylation at $8 \mathrm{~h}$, as compared to the control group (Fig. S1A, B). LF didn't inhibit FAm-induced JNK phosphorylation at $8 \mathrm{~h}$.

\section{Effect of LF on ER stress-induced cytotoxicity and apoptosis}

HepG2 cells were treated with tunicamycin $(2$ and $10 \mu \mathrm{g} / \mathrm{mL})$ to induce ER stress. As shown in Fig. 4A, cell viability was significantly decreased following treatment with tunicamycin at $10 \mu \mathrm{g} / \mathrm{mL}$. In contrast to FAm treatment, LF did not recover cell viability. LDH and caspase-3/7 activities were significantly increased following tunicamycin treatment $(10 \mu \mathrm{g} / \mathrm{mL})$, whereas LF did not inhibit tunicamycin-induced LDH and capsase-3/7 activities (Fig. 4B, C).

\section{Contribution of LF-induced Akt signaling to inhibition of lipotoxicity}

Time-course analysis of Akt phosphorylation following LF treatment showed active Akt phosphorylation until $2 \mathrm{~h}$ after LF treatment (Fig. 5A). A detailed time-course analysis was performed until 30 min after LF treatment to clarify the time of maximum phosphorylation. As shown in Fig. 5B and C, LF induced maximum Akt phosphorylation within 10-20 min, while FAm treatment failed to induce Akt phosphorylation altogether. At this time point, $50 \mu \mathrm{M}$ completely, but $20 \mu \mathrm{M}$ Akt inhibitor VIII only partially abrogated LF-induced Akt phosphorylation (91\% inhibition; Fig. 5D). Therefore, the contribution of LF-induced Akt signaling to the inhibitory effect on FAm-induced lipotoxicity was assessed under conditions of $50 \mu \mathrm{M}$ Akt inhibitor VIII treatment. LF treatment at $100 \mu \mathrm{g} / \mathrm{mL}$ significantly recovered cell viability decreased in the presence of FAm and significantly inhibited FAm-induced LDH and caspase-3/7 activities (Fig. 6A, B and C). Akt inhibitor VIII treatment at $50 \mu \mathrm{M}$ significantly attenuated the effect of LF on LDH activity and abrogated the ones on cell viability and caspase-3/7 activity. 


\section{Discussion}

The results of the present study showed that LF directly inhibited FAm-induced lipotoxicity and recovered cell viability in the HepG2 NAFLD model (Fig. 1A, B, 2A and C), whereas pdLF exhibited no beneficial effect on cell viability (Fig. 1A), suggesting that it was important for LF to reach the liver as an intact without digestion by pepsin. Several studies have reported the distribution of orally administered LF's distribution in rodents. Orally administered LF was detected in many tissues of mice and most abundantly detected in the liver by ELISA method (Fischer et al. 2007). Moreover, it is reported that an 8week regimen of oral LF administration (50-200 $\mathrm{mg} / \mathrm{kg} /$ day) to high-fructose corn syrup-induced NAFLD mice induced a significant increase in LF accumulation in the liver $(14.2 \pm 1.7-22.8 \pm 5.1 \mu \mathrm{g} / \mathrm{g}$ of liver) as well as impairment of NAFLD development ( $\mathrm{Li}$ and Hsieh 2014). In the present study, we clarified that $5 \mu \mathrm{g} / \mathrm{mL}$ LF conveyed a significant beneficial effect against lipotoxicity (LDH and caspase3/7 activities), but not lipid accumulation (Fig. 2A, C and Table. 1). These results suggest that the attenuation of FAm-induced lipotoxicity by LF contributes to impairment of NAFLD development. Besides, LF administration to mice for 4 weeks was reported to significantly decrease triglyceride concentrations in thoracic lymph fluid after feeding, suggesting that LF may inhibit triglyceride absorption by the small intestine (Takeuchi et al. 2004). Taken together, the results of these reports suggest that oral administration of LF can potentially inhibit lipid accumulation as the "first hit" in the liver and impair the "subsequent hit," which promotes NAFLD development.

Regarding cytotoxicity (LDH activity) and apoptosis (caspase-3/7 activity), FAm treatment significantly increased LDH activity at $2 \mathrm{~h}$ and increased caspase-3/7 activity at $4 \mathrm{~h}$, suggesting that cytotoxicity in the early time-course of the experiment was induced by FAm itself (Fig. 3A and B). The inhibitory effect of LF on FAm-induced cytotoxicity until $8 \mathrm{~h}$ after FAm treatment was not as strong as that at $20 \mathrm{~h}$ (Fig. 2A and 3A). Taken together, LF seems to inhibit both of the FAm-induced direct and apoptosis dependent cytotoxicity, but mainly inhibit the latter one. The results that LF completely inhibited FAm-induced caspase-3/7 activity, but not LDH activity (Fig. 3A, C) also support our suggestion. Previous studies 
reported that FAs, especially saturated FAs, such as palmitic acid (C16:0), induce excess production of reactive oxygen species (ROS), which activate signal transduction of both the JNK and ER stress pathways, finally resulting in caspase-3 activation (Cui et al. 2013; Malhi et al. 2006; Nakamura et al. 2009). In the experiment using tunicamycin, LF failed to recover cell viability and inhibit lipotoxicity (Fig. 4A, B and C), strongly suggesting that LF inhibited the JNK signaling pathway, but not ER stressinduced signaling pathway and didn't inhibit caspase-3/7 activity itself by the interaction. Recently, it is Ogasawara et al. reported that LF directly scavenged ROS without a chelating effect (Ogasawara et al. 2014). However, in the present study, LF inhibited caspase-3/7 activity, but appeared not to inhibit JNK phosphorylation (Fig. 3B, Fig. S1), suggesting that the central mechanism of LF was different from that of ROS scavenging.

FA-induced JNK phosphorylation induces the translocation of Bcl family proteins, such as Bim and Bax, to the mitochondria and promotes upregulation of Bim, followed by the release of cytochrome $\mathrm{c}$ from the mitochondria to the cytosol (Eskes et al. 1998; Jin et al. 2006; Lei and Davis 2003; Tsuruta et al. 2004). Excess cytochrome $\mathrm{c}$ in the cytosol forms a complex with the Apaf-1 protein, and the complex cleaves pro-enzyme of caspase-9 into the active form, resulting in activation of caspase-3 (Li et al. 1997). In contrast, phosphorylated Akt induces Ser87 phosphorylation of Bim and Ser184 phosphorylation of Bax, that inhibit translocation of Bim and Bax from the cytosol to the mitochondria by binding them to the 143-3 protein (Gardai et al. 2004; Qi et al. 2006). LF stimulation can induce Akt phosphorylation in several cell types, including HepG2 cells (Lee et al. 2009; Moreno-Navarrete et al. 2009; Xu et al. 2010). Furthermore, LF reportedly inhibits serum deprivation-induced apoptosis in osteoblastic cells via Akt phosphorylation, but not via extracellular signal-regulated kinase (ERK) 1/2 phosphorylation (Grey et al. 2006). Besides, saturated FA itself may induce ERK1/2 phosphorylation (Oh et al. 2014). In the present study, we also confirmed that FAm treatment without LF induced ERK1/2 phosphorylation (data not shown). Therefore, we focused on LF-induced Akt signaling as a possible mechanism of LF activity and performed experimentation to validate this hypothesis. Inhibition experiments using Akt inhibitor VIII clarified that LF-induced Akt signaling contributed to lipotoxicity inhibition (Fig. 6A, B and C), 
suggesting that FAm-induced JNK signal transduction was attenuated by LF via the inhibition of Bim and Bax translocation to mitochondria. Besides, it is well known that activation of the Akt signaling pathway in hepatic cells contributes to recovery from insulin resistance. In fact, Li et al. demonstrated that orally administered LF attenuated high-fructose corn syrup diet-induced insulin resistance in mice (Li and Hsieh 2014). Moreno-navarrete et al. revealed that human LF treatment increased insulin-induced Akt phosphorylation in HepG2 cells (Moreno-Navarrete et al. 2009). These findings support the possibility that LF-induced Akt signaling contributes to the impairment of NAFLD development by the inhibition of both lipotoxicity and insulin resistance. Therefore, it is also important to confirm the effect of LF on FAinduced insulin resistance in this HepG2 NAFLD model in future studies.

In summary, the present study has revealed that LF has a protective effect on FAm-induced lipotoxicity in a HepG2 model of NAFLD and identified the activation of the Akt signaling pathway as a possibly major mechanism.

\section{References}

Avramoglu, R.K., Cianflone, K., and Sniderman, A.D. 1995. Role of the neutral lipid accessible pool in the regulation of secretion of apoB-100 lipoprotein particles by HepG2 cells. J Lipid Res 36(12): 2513-2528.

Chavez-Tapia, N.C., Rosso, N., and Tiribelli, C. 2012. Effect of intracellular lipid accumulation in a new model of non-alcoholic fatty liver disease. BMC Gastroenterol 12: 20. doi: 10.1186/1471-230X-1220.

Cui, W., Ma, J., Wang, X., Yang, W., Zhang, J., and Ji, Q. 2013. Free fatty acid induces endoplasmic reticulum stress and apoptosis of beta-cells by Ca2+/calpain-2 pathways. PLoS One 8(3): e59921. doi: 10.1371/journal.pone.0059921.

Eguchi, Y., Eguchi, T., Mizuta, T., Ide, Y., Yasutake, T., Iwakiri, R., Hisatomi, A., Ozaki, I., Yamamoto, K., Kitajima, Y., Kawaguchi, Y., Kuroki, S., and Ono, N. 2006. Visceral fat accumulation and insulin resistance are important factors in nonalcoholic fatty liver disease. J Gastroenterol 41(5): 
462-469. doi: 10.1007/s00535-006-1790-5.

Eskes, R., Antonsson, B., Osen-Sand, A., Montessuit, S., Richter, C., Sadoul, R., Mazzei, G., Nichols, A., and Martinou, J.C. 1998. Bax-induced cytochrome C release from mitochondria is independent of the permeability transition pore but highly dependent on Mg2+ ions. J Cell Biol 143(1): 217-224.

Fischer, R., Debbabi, H., Blais, A., Dubarry, M., Rautureau, M., Boyaka, P.N., and Tome, D. 2007. Uptake of ingested bovine lactoferrin and its accumulation in adult mouse tissues. Int Immunopharmacol 7(10): 1387-1393. doi: 10.1016/j.intimp.2007.05.019.

Gardai, S.J., Hildeman, D.A., Frankel, S.K., Whitlock, B.B., Frasch, S.C., Borregaard, N., Marrack, P., Bratton, D.L., and Henson, P.M. 2004. Phosphorylation of Bax Ser184 by Akt regulates its activity and apoptosis in neutrophils. J Biol Chem 279(20): 21085-21095. doi: 10.1074/jbc.M400063200.

Gomez-Lechon, M.J., Donato, M.T., Martinez-Romero, A., Jimenez, N., Castell, J.V., and O'Connor, J.E. 2007. A human hepatocellular in vitro model to investigate steatosis. Chem Biol Interact 165(2): 106-116. doi: 10.1016/j.cbi.2006.11.004.

Grey, A., Zhu, Q., Watson, M., Callon, K., and Cornish, J. 2006. Lactoferrin potently inhibits osteoblast apoptosis, via an LRP1-independent pathway. Mol Cell Endocrinol 251(1-2): 96-102. doi: 10.1016/j.mce.2006.03.002.

Harmsen, M.C., Swart, P.J., de Bethune, M.P., Pauwels, R., De Clercq, E., The, T.H., and Meijer, D.K. 1995. Antiviral effects of plasma and milk proteins: lactoferrin shows potent activity against both human immunodeficiency virus and human cytomegalovirus replication in vitro. J Infect Dis 172(2): $380-388$.

Jin, H.O., Park, I.C., An, S., Lee, H.C., Woo, S.H., Hong, Y.J., Lee, S.J., Park, M.J., Yoo, D.H., Rhee, C.H., and Hong, S.I. 2006. Up-regulation of Bak and Bim via JNK downstream pathway in the response to nitric oxide in human glioblastoma cells. J Cell Physiol 206(2): 477-486. doi: $10.1002 / j c p .20488$.

Lee, S.H., Pyo, C.W., Hahm, D.H., Kim, J., and Choi, S.Y. 2009. Iron-saturated lactoferrin stimulates cell cycle progression through PI3K/Akt pathway. Mol Cells 28(1): 37-42. doi: 10.1007/s10059-009- 
0102-3.

Lei, K., and Davis, R.J. 2003. JNK phosphorylation of Bim-related members of the Bcl2 family induces Bax-dependent apoptosis. Proc Natl Acad Sci U S A 100(5): 2432-2437. doi: 10.1073/pnas.0438011100.

Li, P., Nijhawan, D., Budihardjo, I., Srinivasula, S.M., Ahmad, M., Alnemri, E.S., and Wang, X. 1997. Cytochrome c and dATP-dependent formation of Apaf-1/caspase-9 complex initiates an apoptotic protease cascade. Cell 91(4): 479-489.

Li, Y.C., and Hsieh, C.C. 2014. Lactoferrin dampens high-fructose corn syrup-induced hepatic manifestations of the metabolic syndrome in a murine model. PLoS One 9(5): e97341. doi: 10.1371/journal.pone.0097341.

Lin, C.L., Huang, H.C., and Lin, J.K. 2007. Theaflavins attenuate hepatic lipid accumulation through activating AMPK in human HepG2 cells. J Lipid Res 48(11): 2334-2343. doi: 10.1194/jlr.M700128-JLR200.

Malhi, H., Bronk, S.F., Werneburg, N.W., and Gores, G.J. 2006. Free fatty acids induce JNKdependent hepatocyte lipoapoptosis. J Biol Chem 281(17): 12093-12101. doi: 10.1074/jbc.M510660200. Moreno-Navarrete, J.M., Ortega, F.J., Ricart, W., and Fernandez-Real, J.M. 2009. Lactoferrin increases (172Thr)AMPK phosphorylation and insulin-induced (p473Ser)AKT while impairing adipocyte differentiation. Int J Obes (Lond) 33(9): 991-1000. doi: 10.1038/ijo.2009.143.

Morishita, S., Ono, T., Fujisaki, C., Ishihara, Y., Murakoshi, M., Kato, H., Hosokawa, M., Miyashita, K., Sugiyama, K., and Nishino, H. 2013. Bovine lactoferrin reduces visceral fat and liver triglycerides in ICR mice. J Oleo Sci 62(2): 97-103.

Nakamura, S., Takamura, T., Matsuzawa-Nagata, N., Takayama, H., Misu, H., Noda, H., Nabemoto, S., Kurita, S., Ota, T., Ando, H., Miyamoto, K., and Kaneko, S. 2009. Palmitate induces insulin resistance in H4IIEC3 hepatocytes through reactive oxygen species produced by mitochondria. J Biol Chem 284(22): 14809-14818. doi: 10.1074/jbc.M901488200.

Nehra, V., Angulo, P., Buchman, A.L., and Lindor, K.D. 2001. Nutritional and metabolic considerations in the etiology of nonalcoholic steatohepatitis. Dig Dis Sci 46(11): 2347-2352. 
Ogasawara, Y., Imase, M., Oda, H., Wakabayashi, H., and Ishii, K. 2014. Lactoferrin directly scavenges hydroxyl radicals and undergoes oxidative self-degradation: a possible role in protection against oxidative DNA damage. Int J Mol Sci 15(1): 1003-1013. doi: 10.3390/ijms15011003.

Oh, E., Yun, M., Kim, S.K., Seo, G., Bae, J.S., Joo, K., Chae, G.T., and Lee, S.B. 2014. Palmitate induces COX-2 expression via the sphingolipid pathway-mediated activation of NF-kappaB, p38, and ERK in human dermal fibroblasts. Arch Dermatol Res 306(4): 339-345. doi: 10.1007/s00403-0131434-6.

Ono, T., Fujisaki, C., Ishihara, Y., Ikoma, K., Morishita, S., Murakoshi, M., Sugiyama, K., Kato, H., Miyashita, K., Yoshida, T., and Nishino, H. 2013. Potent lipolytic activity of lactoferrin in mature adipocytes. Biosci Biotechnol Biochem 77(3): 566-571.

Ono, T., Morishita, S., Fujisaki, C., Ohdera, M., Murakoshi, M., Iida, N., Kato, H., Miyashita, K., Iigo, M., Yoshida, T., Sugiyama, K., and Nishino, H. 2011. Effects of pepsin and trypsin on the antiadipogenic action of lactoferrin against pre-adipocytes derived from rat mesenteric fat. $\mathrm{Br} \mathrm{J}$ Nutr 105(2): 200-211. doi: 10.1017/S0007114510003259.

Ono, T., Murakoshi, M., Suzuki, N., Iida, N., Ohdera, M., Iigo, M., Yoshida, T., Sugiyama, K., and Nishino, H. 2010. Potent anti-obesity effect of enteric-coated lactoferrin: decrease in visceral fat accumulation in Japanese men and women with abdominal obesity after 8-week administration of enteric-coated lactoferrin tablets. Br J Nutr 104(11): 1688-1695. doi: 10.1017/S0007114510002734.

Qi, X.J., Wildey, G.M., and Howe, P.H. 2006. Evidence that Ser87 of BimEL is phosphorylated by Akt and regulates BimEL apoptotic function. J Biol Chem 281(2): 813-823. doi: 10.1074/jbc.M505546200.

Sekine, K., Ushida, Y., Kuhara, T., Iigo, M., Baba-Toriyama, H., Moore, M.A., Murakoshi, M., Satomi, Y., Nishino, H., Kakizoe, T., and Tsuda, H. 1997. Inhibition of initiation and early stage development of aberrant crypt foci and enhanced natural killer activity in male rats administered bovine lactoferrin concomitantly with azoxymethane. Cancer Lett 121(2): 211-216.

Shoji, H., Oguchi, S., Shinohara, K., Shimizu, T., and Yamashiro, Y. 2007. Effects of ironunsaturated human lactoferrin on hydrogen peroxide-induced oxidative damage in intestinal 
epithelial cells. Pediatr Res 61(1): 89-92. doi: 10.1203/01.pdr.0000250198.22735.20.

Takeuchi, T., Shimizu, H., Ando, K., and Harada, E. 2004. Bovine lactoferrin reduces plasma triacylglycerol and NEFA accompanied by decreased hepatic cholesterol and triacylglycerol contents in rodents. Br J Nutr 91(4): 533-538. doi: 10.1079/BJN20041090.

Tilg, H., and Moschen, A.R. 2010. Evolution of inflammation in nonalcoholic fatty liver disease: the multiple parallel hits hypothesis. Hepatology 52(5): 1836-1846. doi: 10.1002/hep.24001.

Tomita, M., Bellamy, W., Takase, M., Yamauchi, K., Wakabayashi, H., and Kawase, K. 1991. Potent antibacterial peptides generated by pepsin digestion of bovine lactoferrin. J Dairy Sci 74(12): 41374142. doi: 10.3168/jds.S0022-0302(91)78608-6.

Tsuruta, F., Sunayama, J., Mori, Y., Hattori, S., Shimizu, S., Tsujimoto, Y., Yoshioka, K., Masuyama, N., and Gotoh, Y. 2004. JNK promotes Bax translocation to mitochondria through phosphorylation of 14-3-3 proteins. EMBO J 23(8): 1889-1899. doi: 10.1038/sj.emboj.7600194.

Wu, X., Zhang, L., Gurley, E., Studer, E., Shang, J., Wang, T., Wang, C., Yan, M., Jiang, Z., Hylemon, P.B., Sanyal, A.J., Pandak, W.M., Jr., and Zhou, H. 2008. Prevention of free fatty acid-induced hepatic lipotoxicity by 18beta-glycyrrhetinic acid through lysosomal and mitochondrial pathways. Hepatology 47(6): 1905-1915. doi: 10.1002/hep.22239.

Xu, X.X., Jiang, H.R., Li, H.B., Zhang, T.N., Zhou, Q., and Liu, N. 2010. Apoptosis of stomach cancer cell SGC-7901 and regulation of Akt signaling way induced by bovine lactoferrin. J Dairy Sci 93(6): 2344-2350. doi: 10.3168/jds.2009-2926.

Yagi, M., Suzuki, N., Takayama, T., Arisue, M., Kodama, T., Yoda, Y., Numasaki, H., Otsuka, K., and Ito, K. 2008. Lactoferrin suppress the adipogenic differentiation of MC3T3-G2/PA6 cells. J Oral Sci 50(4): 419-425.

Zhang, J., Zhao, Y., Xu, C., Hong, Y., Lu, H., Wu, J., and Chen, Y. 2014. Association between serum free fatty acid levels and nonalcoholic fatty liver disease: a cross-sectional study. Sci Rep 4: 5832. doi: 10.1038/srep05832.

Zimecki, M., Wlaszczyk, A., Cheneau, P., Brunel, A.S., Mazurier, J., Spik, G., and Kubler, A. 1998. Immunoregulatory effects of a nutritional preparation containing bovine lactoferrin taken orally by 
healthy individuals. Arch Immunol Ther Exp (Warsz) 46(4): 231-240. 
Tables

Table 1. Effect of LF on intracellular lipid accumulation

\begin{tabular}{lcc}
\hline \multicolumn{1}{c}{ Group } & $\begin{array}{c}\text { Intracellular Triglyceride } \\
(\mu \mathrm{g} / \mathrm{mg} \text {-protein })\end{array}$ & $* p$ \\
\hline Control & $228 \pm 45$ & $<0.001$ \\
FAm $1 \mathrm{mM}$ & $1095 \pm 58$ & - \\
FAm $1 \mathrm{mM}+$ LF $100 \mu \mathrm{g} / \mathrm{mL}$ & $1038 \pm 74$ & $\mathrm{~N} . \mathrm{S}$ \\
\hline${ }^{*} p<0.05$; Statistical analysis was performed using the Dunnett test, compared with FAm $(1 \mathrm{mM})$.
\end{tabular}


Figure captions and legends

Fig. 1. Effects of lactoferrin (LF) on cell viability and lipid accumulation in the FA mixture (FAm)induced NAFLD model. (A) LF (100 $\mu \mathrm{g} / \mathrm{mL})$ and pepsin-digested LF (pdLF) $(100 \mu \mathrm{g} / \mathrm{mL})$ were added to HepG2 cells with FAm (1 mM) for $20 \mathrm{~h}$ and resazurin reduction activity as the outcome of cell viability was analyzed. (B) LF $(5,20$, and $100 \mu \mathrm{g} / \mathrm{mL})$ was added to HepG2 cells with FAm (1 mM) for $20 \mathrm{~h}$ and resazurin reduction activity was analyzed. All data are expressed as means \pm standard deviations, $\mathrm{n}=4$. Statistical analysis was performed by one-way ANOVA and a post-hoc Tukey-Kramer test $(p<0.05)$. Different letters indicate statistical significance between groups. At $20 \mathrm{~h}$ after FAm and LF treatment, microscopic observation was performed by phase-contrast microscopy. (C) no-treated, (D) FAm (1 mM) and (E) FAm $(1 \mathrm{mM})+\mathrm{LF}(100 \mu \mathrm{g} / \mathrm{mL})$.

Fig. 2. Effects of LF on FAm-induced lipotoxicity. LF (5, 20, and $100 \mu \mathrm{g} / \mathrm{mL})$ or SP600125 (50 $\mu \mathrm{g} / \mathrm{mL})$ was added to HepG2 cells with FAm for $20 \mathrm{~h}$ and lipotoxicity-related outcomes were analyzed. (A) and (B) Lactic dehydrogenase (LDH) activity in culture medium. (C) and (D) Caspase-3/7 activity in cells. (A) and (C) All data are expressed as means \pm standard deviations, $n=4$. (B) and (D) All data are expressed as means \pm standard deviations, $n=3$. Statistical analysis was performed by one-way ANOVA and a post-hoc Tukey-Kramer test $(p<0.05)$. Different letters indicate statistical significance between groups.

Fig. 3. Effects of LF on FAm-induced lipotoxicity in early time-points. LF (100 $\mu \mathrm{g} / \mathrm{mL})$ was added to HepG2 cells with FAm $(1 \mathrm{mM})$ and lipotoxicity-related outcomes were analyzed at 2, 4, and $8 \mathrm{~h}$ after LF treatment. (A) LDH activity in culture medium .(B) Caspase-3/7 activity in cells. Most groups are $\mathrm{n}=5$ except for the FAm-treated group at $8 \mathrm{~h}$ in LDH analysis $(\mathrm{n}=4)$. All data are expressed as means \pm standard deviations. Statistical analysis was performed by one-way ANOVA and a post-hoc Student t-test and Bonferroni corrections $(p<0.05)$. 
Fig. 4. Effects of LF on endoplasmic reticulum (ER)-stress induced lipotoxicity. LF $(100 \mu \mathrm{g} / \mathrm{mL})$ was added to HepG2 cells with tunicamycin $(10 \mu \mathrm{g} / \mathrm{mL})$ for $20 \mathrm{~h}$ and lipotoxicity-related outcomes were analyzed. (A) Cell viability. (B) LDH activity in culture medium. (C) Caspase 3/7 activity. All data are presented as means \pm standard deviations, $\mathrm{n}=4$. Statistical analysis was performed using one-way ANOVA and a post-hoc Tukey-Kramer test $(p<0.05)$. Different letters indicate statistical significance between groups.

Fig. 5. Effect of Akt Inhibitor VIII on Akt phosphorylation in a LF-treated HepG2 NAFLD model. LF $(100 \mu \mathrm{g} / \mathrm{mL})$ was added to HepG2 cells with FAm $(1 \mathrm{mM})$ and cells were lysed at various times. Two samples in each group were pooled and phospho-Akt and total-Akt were analyzed by western blotting. (A) Time-course Akt phosphorylation (0-6 h). (B) Detailed time-course of Akt phosphorylation (0-30 min). (C) HepG2 cells were treated with FAm with or without LF. Akt phosphorylation was analyzed at 20 min after treatment $(\mathrm{n}=2)$. (D) Akt Inhibitor VIII $(20-100 \mu \mathrm{M})$ was simultaneously added to HepG2 cells with LF and FAm. Akt phosphorylation was analyzed at $20 \mathrm{~min}$ after treatment. The ratio of phospho-Akt/total-Akt was determined using a light-capture instrument.

Fig. 6. The contribution of Akt signaling to the inhibitory effect of LF on FAm-induced lipotoxicity. HepG2 cells were treated with FAm $(1 \mathrm{mM})$, LF $(100 \mu \mathrm{g} / \mathrm{mL})$, and Akt Inhibitor VIII $(50 \mu \mathrm{M})$ for $2.5 \mathrm{~h}$. Then, Akt Inhibitor VIII was removed by changing the medium and the cells were cultured with FAm (1 $\mathrm{mM})$ and LF (100 $\mu \mathrm{g} / \mathrm{mL})$ for $17.5 \mathrm{~h}$. Afterward, lipotoxicity-related outcomes were analyzed. (A) Cell viability. (B) LDH activity in culture medium. (C) Caspase-3/7 activity in cells. All data are expressed as means \pm standard deviations, $n=4$. Statistical analysis was performed by one-way ANOVA and a posthoc Tukey-Kramer test $(p<0.05)$. Different letters indicate statistical significance between groups. 
Supplemental Fig. S1. Effects of LF on FAm-induced c-jun-N-terminal kinase (JNK) phosphorylation. LF $(100 \mu \mathrm{g} / \mathrm{mL})$ was added to HepG2 cells with $1 \mathrm{mM}$ FAm and cells were lysed at $8 \mathrm{~h}$. (A) Three samples in each group were prepared and phospho-JNK and total-JNK were analyzed by western blotting. (B) The ratio of phospho-JNK/total-JNK was determined using a light-capture instrument. 
Figures

Fig. 1. Effects of lactoferrin (LF) on cell viability and lipid accumulation in the FA mixture (FAm)induced NAFLD model.

A

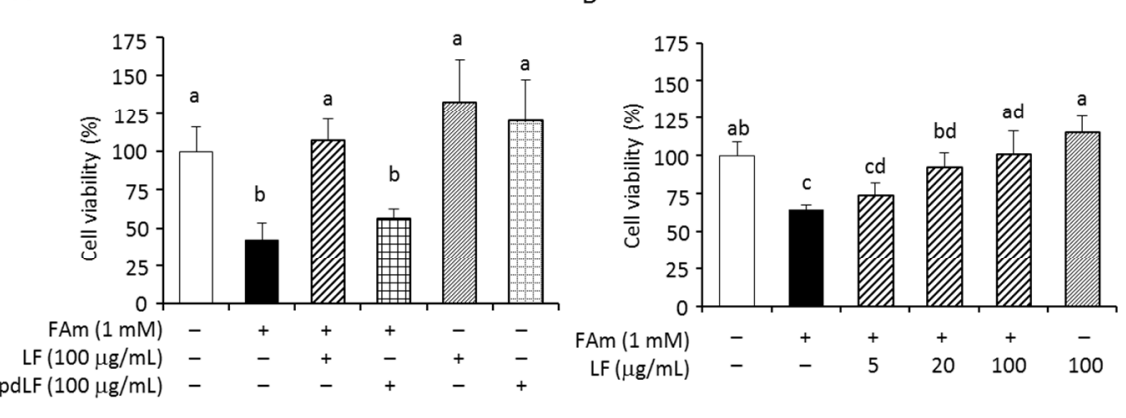

C

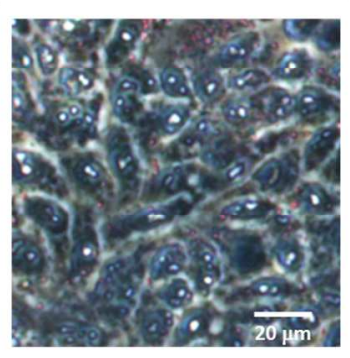

D

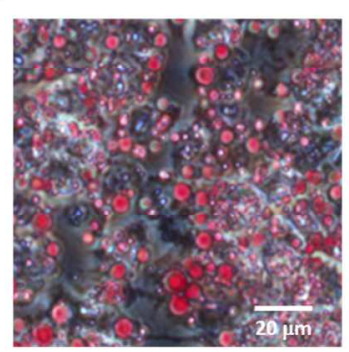

E

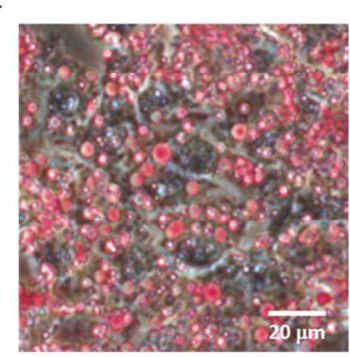

Fig. 2. Effects of LF on FAm-induced lipotoxicity.

A

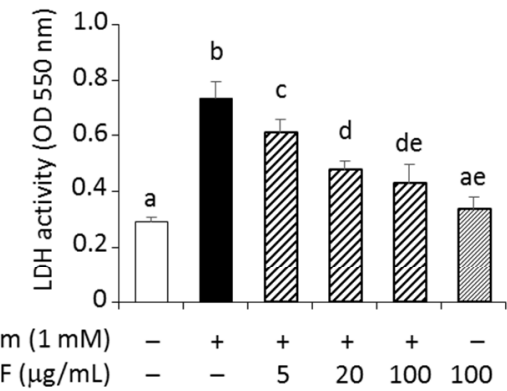

C

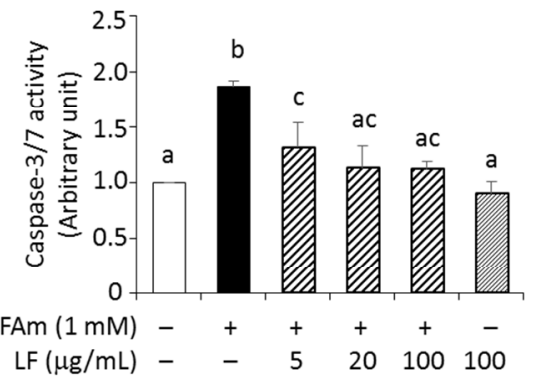

B

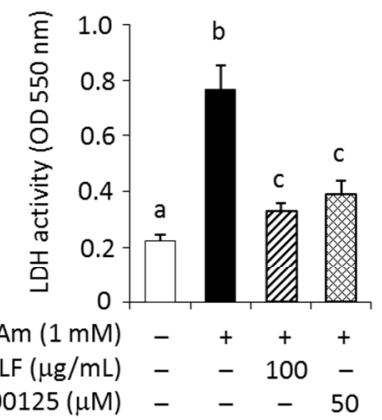

D

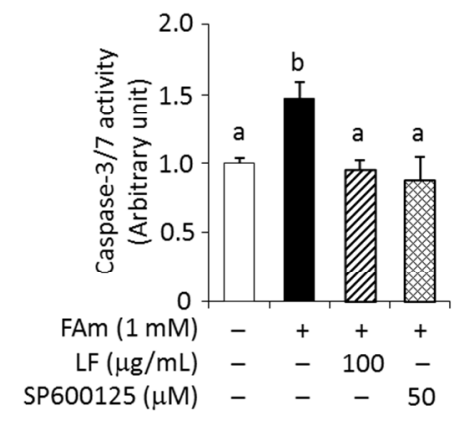


Fig. 3. Effects of LF on FAm-induced lipotoxicity in early time-points

A

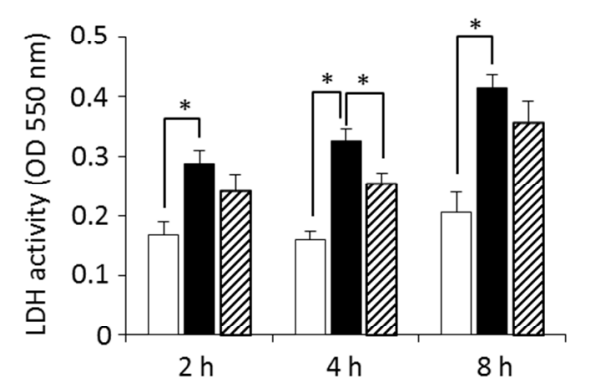

No-treated
B

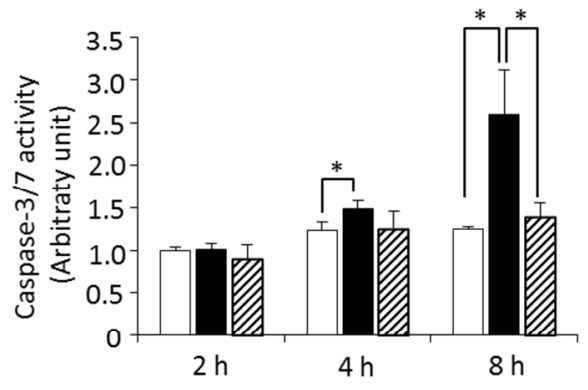

FAm $(1 \mathrm{mM}) \mathbb{Z}$ FAm $(1 \mathrm{mM})+\mathrm{LF}(100 \mu \mathrm{g} / \mathrm{mL})$

Fig. 4. Effects of LF on endoplasmic reticulum (ER)-stress induced lipotoxicity.

A

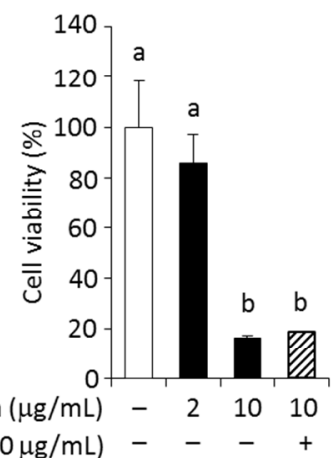

B

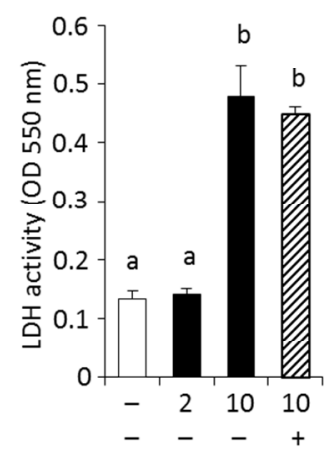

C

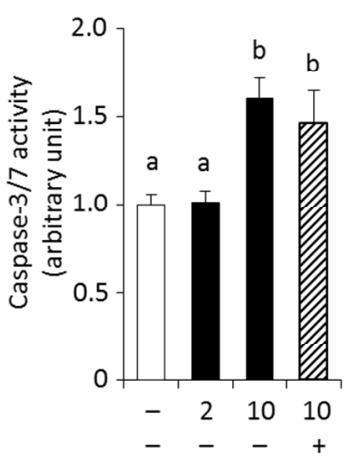

Fig. 5. Effect of Akt inhibitor VIII on Akt phosphorylation in a LF-treated HepG2 NAFLD model.

A

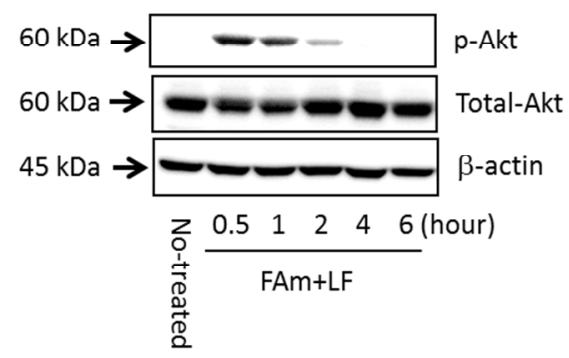

C

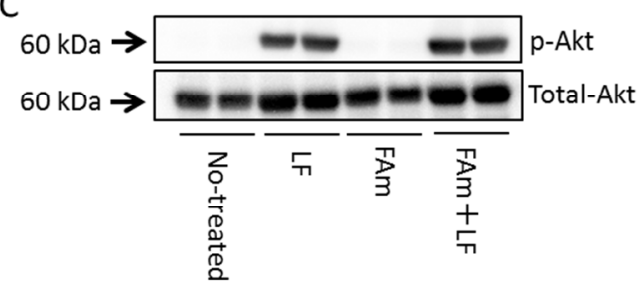

B

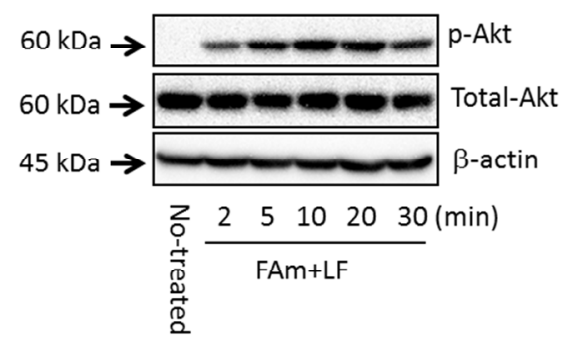

D

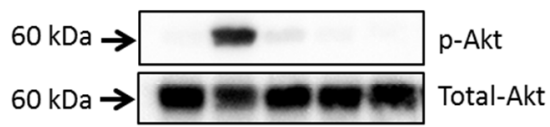

Inhibitory rate (\%) - 091100100

FAm $(1 \mathrm{mM})-++++$

$\mathrm{LF}(100 \mu \mathrm{g} / \mathrm{mL})-++++$

Akt Inhibitor VIII $(\mu \mathrm{M}) \quad-\quad-2050100$ 
Fig. 6. The contribution of Akt signaling to the inhibitory effect of LF on FAm-induced lipotoxicity.

A

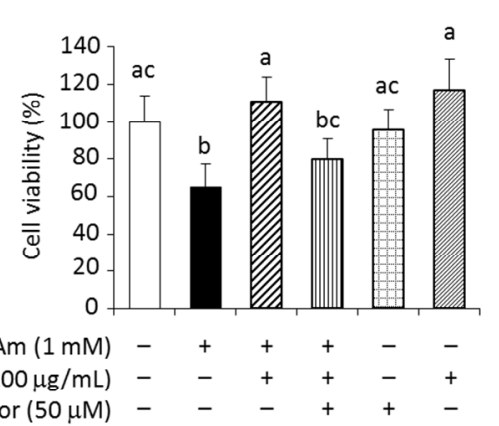

B

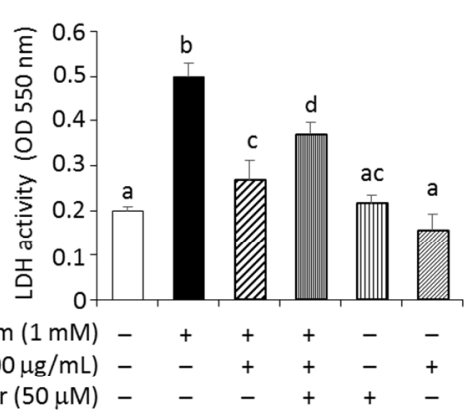

C

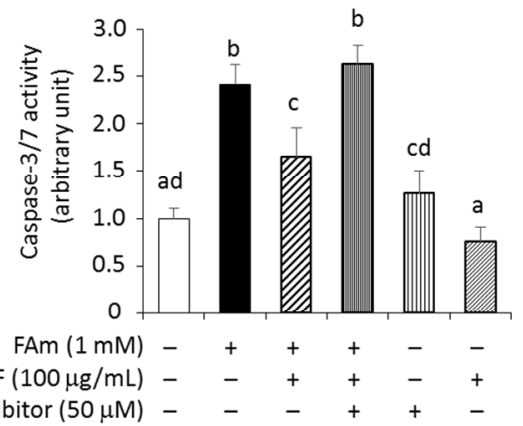

Supplemental Fig. S1. Effects of LF on FAm-induced c-jun-N-terminal kinase (JNK) phosphorylation.

A

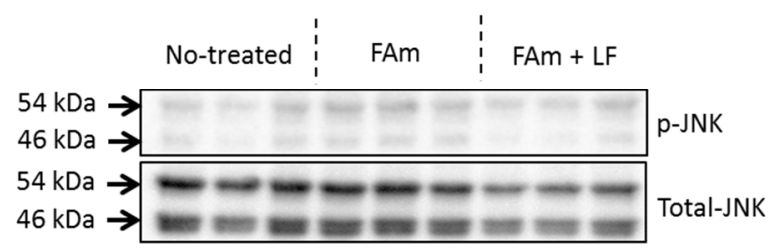

B

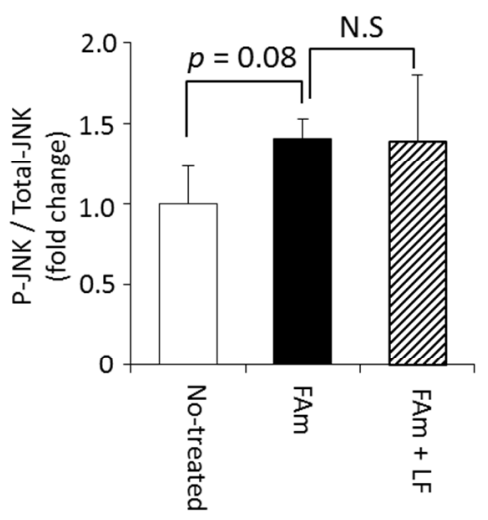

